\title{
Functional consequences of non-equilibrium dynamics caused by antisymmetric and symmetric learning rules
}

\author{
Dmytro Grytskyy ${ }^{1 *}$, Markus Diesmann ${ }^{1,2,3}$, Moritz Helias ${ }^{1}$ \\ From 24th Annual Computational Neuroscience Meeting: CNS*2015 \\ Prague, Czech Republic. 18-23 July 2015
}

Connectivity in the brain is asymmetric, which is evident by Dale's principle of excitatory and inhibitory neurons. As a consequence, biologically realistic neuronal networks cannot be in thermodynamic equilibrium [1]. Even in a stationary state, probability fluxes perpetuate, leading to non-equilibrium steady states $[2,3]$. We here investigate the computational consequences of nonequilibrium dynamics for synaptic plasticity and learning. Formulating the stochastic dynamics in sparsely connected networks of non-linear Langevin equations in terms of path integrals [4], we show that biologically plausible correlation-sensitive plasticity rules follow from first principles. We investigate two different rules: 1.) Maximizing a measure of irreversibility [2] by gradient descent with respect to the weights, we obtain a local learning rule sensitive to the derivative of the covariance between the pre- and postsynaptic neuron. The obtained rule can be interpreted as spike-timing dependent plasticity (STDP) $[5,6]$ with a narrow antisymmetric learning window. We show that the learning rule increases synaptic weights in the direction of the (direct or indirect) causal influence between a pair of neurons. In this way indirect causal relationships are transformed into strengthened direct connections. An instability of the state with vanishing weights manifests itself as spontaneous symmetry breaking and the divergence of connection weights. 2.) We investigate a rule that is sensitive to the zero-time-lag covariance. It can be considered as a complement to the first rule approximating STDP with a symmetric narrow learning window. This rule results in the strengthening of loops, of mutual

\footnotetext{
* Correspondence: d.grytskyy@fz-juelich.de

1 Institute of Neuroscience and Medicine (INM-6) and Institute for Advanced Simulation (IAS-6), Jülich Research Centre and JARA, Jülich, Germany Full list of author information is available at the end of the article
}

connections between neurons getting common input, and of indirect (also backward) connections between neurons $\mathrm{a}$ and $\mathrm{b}$ if a influences $\mathrm{b}$. We derive analytical expressions that describe these effects quantitatively. We show how nonlinearities in the neuronal transmission naturally stabilize synaptic weights and how the limited dynamic range of neuronal activity mediates competition between synapses. The work elucidates the tight connection between measures of reversible and irreversible dynamics and the structures resulting from learning rules that optimize either of the two.

\section{Acknowledgements}

Partially supported by the Helmholtz portfolio theme SMHB, the Jülich Aachen Research Alliance (JARA), HGF Young Investigator's Group VH-NG1028, EU Grants 269921 (BrainScaleS) and 604102 (HBP).

\section{Authors' details}

${ }^{1}$ Institute of Neuroscience and Medicine (INM-6) and Institute for Advanced Simulation (IAS-6), Jülich Research Centre and JARA, Jülich, Germany. ${ }^{2}$ Department of Psychiatry, Psychotherapy and Psychosomatics, Medical Faculty, RWTH Aachen University, Aachen, Germany. ${ }^{3}$ Department of Physics, Faculty 1, RWTH Aachen University, Aachen, Germany.

\section{Published: 18 December 2015}

\section{References}

1. Sompolinsky H: Statistical mechanics of neuronal networks. Physics Today 1988, 70-80.

2. Seifert U: Stochastic thermodynamics, fluctuation theorems, and molecular machines. Rep Prog Phys 2012, 75:126001, doi:10.1088/00344885/75/12/126001.

3. Evans DJ, Morriss GP: Statistical Mechanics of Non Equilibrium Liquids London: Academic Press; 1990.

4. Wio HS, Colet P, San Miguel M: Path-integral formulation for stochastic processes driven by colored noise. Phys Rev A 1989, 40:7312-7324.

5. Bi GQ, Poo MM: Synaptic modifications in cultured Hippocampal neurons: dependence on spike timing, synaptic strength, and postsynaptic cell type. J Neurosci 1998, 18:10464-72. 
6. Morrison A, Diesmann M, Gerstner W: Phenomenological models of synaptic plasticity based on spike timing. Biol Cybern 2008, 98:459-478.

doi:10.1186/1471-2202-16-S1-P96

Cite this article as: Grytskyy et al:: Functional consequences of non-

equilibrium dynamics caused by antisymmetric and symmetric learning rules. BMC Neuroscience 2015 16(Suppl 1):P96.

Submit your next manuscript to BioMed Central and take full advantage of:

- Convenient online submission

- Thorough peer review

- No space constraints or color figure charges

- Immediate publication on acceptance

- Inclusion in PubMed, CAS, Scopus and Google Scholar

- Research which is freely available for redistribution

Submit your manuscript at www.biomedcentral.com/submit

() BioMed Central 